\title{
Erratum: Feynman rules for the rational part of the Electroweak 1-loop amplitudes
}

\author{
M.V. Garzelli, ${ }^{a, b}$ I. Malamos ${ }^{c}$ and R. Pittau ${ }^{a}$ \\ ${ }^{a}$ Departamento de Física Teórica y del Cosmos y CAFPE, Universidad de Granada, \\ E-18071 Granada, Spain \\ ${ }^{b}$ INFN Milano, \\ I-20133 Milano, Italy \\ ${ }^{c}$ Department of Theoretical High Energy Physics, Institute for Mathematics, \\ Astrophysics and Particle Physics, Radboud Universiteit Nijmegen, \\ 6525 AJ Nijmegen, the Netherlands \\ E-mail: garzelli@to.infn.it, J.Malamos@science.ru.nl, pittau@ugr.es
}

ERRATUM TO: JHEP01(2010)040

ABSTRACT: We present the complete set of Feynman rules producing the rational terms of kind $\mathrm{R}_{2}$ needed to perform any 1-loop calculation in the Electroweak Standard Model. Our results are given both in the 't Hooft-Veltman and in the Four Dimensional Helicity regularization schemes. We also verified, by using both the 't Hooft-Feynman gauge and the Background Field Method, a huge set of Ward identities -up to 4-points- for the complete rational part of the Electroweak amplitudes. This provides a stringent check of our results and, as a by-product, an explicit test of the gauge invariance of the Four Dimensional Helicity regularization scheme in the complete Standard Model at 1-loop. The formulae presented in this paper provide the last missing piece for completely automatizing, in the framework of the OPP method, the 1-loop calculations in the $\mathrm{SU}(3) \times \mathrm{SU}(2) \times \mathrm{U}(1)$ Standard Model.

By comparing with an independent computation we performed in a general $R_{\xi}$ gauge, and thanks to Huasheng Shao, that recomputed, independently of us, all of the $\mathrm{R}_{2}$ effective vertices in the 't Hooft-Feynman gauge, we found a few problems in our formulae. We would like to correct them here. 
The vertex $A l \bar{l}$ in eq. (3.6) should read

$$
\begin{aligned}
A l \bar{l}: C_{-}= & \frac{1}{4}\left[\frac{\left(1+\lambda_{H V}\right) Q_{l}^{3}}{4 c_{w}^{2}}+\frac{m_{l}^{2}}{8 s_{w}^{2} m_{W}^{2}}\left(\frac{Q_{l}}{4}+Q_{l} I_{3 l}^{2}\right)\right] \\
C_{+}= & \frac{1}{4}\left[\frac{\left(1+\lambda_{H V}\right) Q_{l}^{3}}{4 c_{w}^{2}}-\frac{\left(1+\lambda_{H V}\right) Q_{l}^{2} I_{3 l}}{2 c_{w}^{2}}+\frac{\left(1+\lambda_{H V}\right) Q_{l} I_{3 l}^{2}}{4 s_{w}^{2} c_{w}^{2}}\right. \\
& \left.+\frac{1}{4 s_{w}^{2}}\left(\frac{m_{l}^{2} Q_{l}\left(1+4 I_{3 l}^{2}\right)}{8 m_{W}^{2}}-\frac{\left(1+\lambda_{H V}\right)}{2}\right)\right] .
\end{aligned}
$$

The vertex $Z \phi^{+} \phi^{-}$in eq. (3.8) should read

$$
\begin{aligned}
Z \phi^{+} \phi^{-}: C= & \frac{i}{48 s_{w} c_{w}}\left\{\frac{1-24 c_{w}^{4}}{16 c_{w}^{2} s_{w}^{2}}+\frac{1}{m_{W}^{2}}\left(-\sum_{i=1}^{3}\left(m_{e_{i}}^{2}\left(Q_{e_{i}}+\frac{I_{3 \nu_{i}}}{s_{w}^{2}}\right)\right)\right.\right. \\
& \left.\left.+N_{\text {col }} \sum_{i, j=1}^{3}\left(V_{u_{i} d_{j}} V_{d_{j} u_{i}}^{\dagger}\left[\left(m_{u_{i}}^{2}+m_{d_{j}}^{2}\right)+\frac{m_{u_{i}}^{2} I_{3 d_{j}}-m_{d_{i}}^{2} I_{3 u_{i}}}{s_{w}^{2}}\right]\right)\right)\right\} .
\end{aligned}
$$

Eq. (3.16) should be replaced by

$$
\begin{aligned}
& \left.\begin{array}{l}
H \chi A A \\
H \chi A Z \\
H \chi Z Z \\
H \chi W^{+} W^{-}
\end{array}\right\}: C=0
\end{aligned}
$$

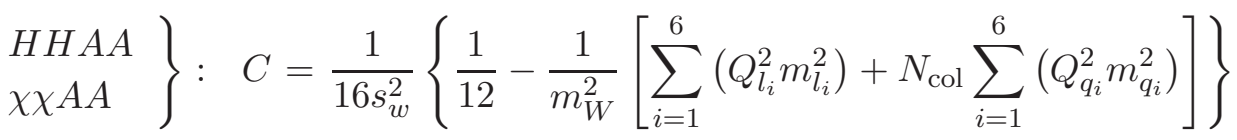

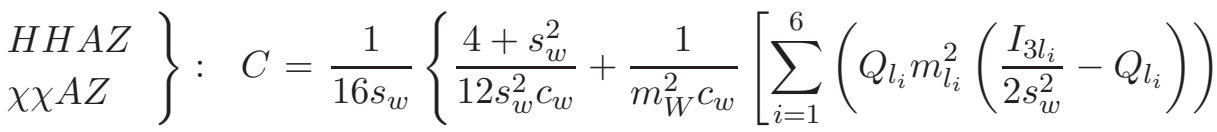

$$
\begin{aligned}
& \left.\left.+N_{\mathrm{col}} \sum_{i=1}^{6}\left(Q_{q_{i}} m_{q_{i}}^{2}\left(\frac{I_{3 q_{i}}}{2 s_{w}^{2}}-Q_{q_{i}}\right)\right)\right]\right\}
\end{aligned}
$$

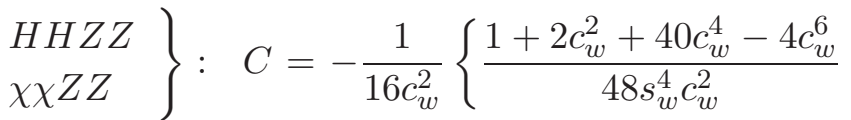

$$
\begin{aligned}
& +\frac{1}{m_{W}^{2}}\left[\sum_{i=1}^{6}\left(m_{l_{i}}^{2}\left(Q_{l_{i}}^{2}+\frac{4 I_{3 l_{i}}^{2}}{3 s_{w}^{4}}-\frac{Q_{l_{i}} I_{3 l_{i}}}{s_{w}^{2}}\right)\right)\right. \\
& \left.\left.+N_{\mathrm{col}} \sum_{i=1}^{6}\left(m_{q_{i}}^{2}\left(Q_{q_{i}}^{2}+\frac{4 I_{3 q_{i}}^{2}}{3 s_{w}^{4}}-\frac{Q_{q_{i}} I_{3 q_{i}}}{s_{w}^{2}}\right)\right)\right]\right\} \\
& \left.\begin{array}{l}
H H W^{-} W^{+} \\
\chi \chi W^{-} W^{+}
\end{array}\right\}: C=-\frac{1}{48 s_{w}^{4}}\left\{\frac{1+38 c_{w}^{2}}{16 c_{w}^{2}}\right. \\
& \left.+\frac{1}{m_{W}^{2}}\left[\sum_{i=1}^{3} m_{e_{i}}^{2}+N_{\mathrm{col}} \sum_{i, j=1}^{3}\left(V_{u_{i} d_{j}} V_{d_{j} u_{i}}^{\dagger}\left(m_{u_{i}}^{2}+m_{d_{j}}^{2}\right)\right)\right]\right\}
\end{aligned}
$$




$$
\begin{aligned}
& \left.\begin{array}{l}
H \phi^{+} W^{-} A \\
\phi^{-} H A W^{+}
\end{array}\right\}: C=K_{1} \\
& \chi \phi^{+} W^{-} A \quad: \quad C=-i K_{1} \\
& \phi^{-} \chi A W^{+} \quad: \quad C=i K_{1} \\
& \left.\begin{array}{l}
H \phi^{+} W^{-} Z \\
\phi^{-} H Z W^{+}
\end{array}\right\}: C=K_{2} \\
& \chi \phi^{+} W^{-} Z \quad: \quad C=-i K_{2} \\
& \phi^{-} \chi Z W^{+}: C=i K_{2} \\
& \phi^{-} \phi^{+} A A \quad: \quad C=-\frac{1}{12 s_{w}^{2}}\left\{\frac{1+21 c_{w}^{2}}{16 c_{w}^{2}}+\frac{1}{m_{W}^{2}}\left[\sum_{i=1}^{3} m_{e_{i}}^{2}\right.\right. \\
& \left.\left.+\frac{5}{6} N_{\mathrm{col}} \sum_{i, j=1}^{3}\left(V_{u_{i} d_{j}} V_{d_{j} u_{i}}^{\dagger}\left(m_{u_{i}}^{2}+m_{d_{j}}^{2}\right)\right)\right]\right\} \\
& \phi^{-} \phi^{+} A Z \quad: \quad C=\frac{1}{12 s_{w} c_{w}}\left\{\frac{42 c_{w}^{4}-10 c_{w}^{2}-1}{32 s_{w}^{2} c_{w}^{2}}\right. \\
& -\frac{1}{m_{W}^{2}}\left[\sum_{i=1}^{3}\left(m_{e_{i}}^{2} Q_{e_{i}}\left(Q_{e_{i}}+\frac{5}{8} \frac{I_{3 \nu_{i}}}{s_{w}^{2}}\right)\right)\right. \\
& +N_{\mathrm{col}} \sum_{i, j=1}^{3}\left[V _ { u _ { i } d _ { j } } V _ { d _ { j } u _ { i } } ^ { \dagger } \left(m_{u_{i}}^{2}\left(\frac{5}{6}-\frac{I_{3 d_{i}}}{s_{w}^{2}}\left(Q_{d_{j}}-\frac{5}{8} Q_{u_{i}}\right)\right)\right.\right. \\
& \left.\left.\left.\left.+m_{d_{j}}^{2}\left(\frac{5}{6}-\frac{I_{3 u_{i}}}{s_{w}^{2}}\left(Q_{u_{i}}-\frac{5}{8} Q_{d_{j}}\right)\right)\right)\right]\right]\right\} \\
& \phi^{-} \phi^{+} Z Z \quad: \quad C=\frac{1}{12 c_{w}^{2}}\left\{\frac{-1+2 c_{w}^{2}+44 c_{w}^{4}-84 c_{w}^{6}}{64 s_{w}^{4} c_{w}^{2}}\right. \\
& -\frac{1}{m_{W}^{2}}\left[\sum_{i=1}^{3}\left(m_{e_{i}}^{2}\left(Q_{e_{i}}^{2}+\frac{5}{4} \frac{Q_{e_{i}} I_{3 \nu_{i}}}{s_{w}^{2}}+\frac{I_{3 \nu_{i}}^{2}}{s_{w}^{4}}\right)\right)\right. \\
& +N_{\mathrm{col}} \sum_{i, j=1}^{3}\left[V _ { u _ { i } d _ { j } } V _ { d _ { j } u _ { i } } ^ { \dagger } \left(m_{u_{i}}^{2}\left(\frac{5}{6}-\frac{I_{3 d_{i}}}{s_{w}^{2}}\left(2 Q_{d_{j}}-\frac{5}{4} Q_{u_{i}}\right)+\frac{I_{3 d_{i}}^{2}}{s_{w}^{4}}\right)\right.\right. \\
& \left.\left.\left.\left.+m_{d_{j}}^{2}\left(\frac{5}{6}-\frac{I_{3 u_{i}}}{s_{w}^{2}}\left(2 Q_{u_{i}}-\frac{5}{4} Q_{d_{j}}\right)\right)+\frac{I_{3 u_{i}}^{2}}{s_{w}^{4}}\right)\right]\right]\right\}
\end{aligned}
$$




$$
\begin{aligned}
\phi^{-} \phi^{+} W^{-} W^{+}: C= & -\frac{1}{48 s_{w}^{4}}\left\{\frac { 1 } { m _ { W } ^ { 2 } } \left[\left(\sum_{i=1}^{3} m_{e_{i}}^{2}\right.\right.\right. \\
& \left.\left.+N_{\text {col }} \sum_{i, j, k, l=1}^{3}\left(V_{u_{i} d_{j}} V_{d_{j} u_{k}}^{\dagger} V_{u_{k} d_{l}} V_{d_{l} u_{i}}^{\dagger}\left(m_{u_{i}} m_{u_{k}}+m_{d_{j}} m_{d_{l}}\right)\right)\right)\right] \\
& \left.+\frac{38 c_{w}^{2}+1}{16 c_{w}^{2}}\right\} .
\end{aligned}
$$

Eq. (3.17) should be replaced by

$$
\begin{aligned}
K_{1} & =\frac{1}{24 s_{w}^{3}}\left\{\frac{1+22 c_{w}^{2}}{32 c_{w}^{2}}+K\right\} \\
K_{2} & =\frac{1}{24 s_{w}^{2} c_{w}}\left\{\frac{1+21 c_{w}^{2}-22 c_{w}^{4}}{32 c_{w}^{2} s_{w}^{2}}+K\right\} \\
K & =\frac{1}{8 m_{W}^{2}}\left[\sum_{i=1}^{3} m_{e_{i}}^{2}+N_{\mathrm{col}} \sum_{i, j=1}^{3}\left(V_{u_{i} d_{j}} V_{d_{j} u_{i}}^{\dagger}\left(3 m_{d_{j}}^{2}+2 m_{u_{i}}^{2}\right)\right)\right] .
\end{aligned}
$$

Eq. (3.18) should be replaced by

$$
\begin{aligned}
u \bar{u}: C_{-}= & \frac{1}{16}\left[\left(1+\lambda_{H V}\right) \frac{Q_{u}^{2}}{c_{w}^{2}}+\frac{m_{u}^{2}}{2 s_{w}^{2} m_{W}^{2}}\left(\frac{1}{2} \sum_{j=1}^{3}\left(V_{u d_{j}} V_{d_{j} u}^{\dagger}\right)+\frac{1}{4}+I_{3 u}^{2}\right)\right] \\
C_{+}= & \frac{1}{16}\left[\left(1+\lambda_{H V}\right)\left(\frac{1}{c_{w}^{2}}\left(Q_{u}^{2}+\frac{I_{3 u}^{2}}{s_{w}^{2}}-2 Q_{u} I_{3 u}\right)+\frac{1}{2 s_{w}^{2}} \sum_{j=1}^{3}\left(V_{u d_{j}} V_{d_{j} u}^{\dagger}\right)\right)\right. \\
& \left.+\frac{1}{2 m_{W}^{2} s_{w}^{2}}\left(\frac{1}{2} \sum_{j=1}^{3}\left(V_{u d_{j}} V_{d_{j} u}^{\dagger} m_{d_{j}}^{2}\right)+m_{u}^{2}\left(\frac{1}{4}+I_{3 u}^{2}\right)\right)\right] \\
d \bar{d}: C_{-}= & \frac{1}{16}\left[\left(1+\lambda_{H V}\right) \frac{Q_{d}^{2}}{c_{w}^{2}}+\frac{m_{d}^{2}}{2 s_{w}^{2} m_{W}^{2}}\left(\frac{1}{2} \sum_{i=1}^{3}\left(V_{u_{i} d} V_{d u_{i}}^{\dagger}\right)+\frac{1}{4}+I_{3 d}^{2}\right)\right] \\
C_{+}= & \frac{1}{16}\left[\left(1+\lambda_{H V}\right)\left(\frac{1}{c_{w}^{2}}\left(Q_{d}^{2}+\frac{I_{3 d}^{2}}{s_{w}^{2}}-2 Q_{d} I_{3 d}\right)+\frac{1}{2 s_{w}^{2}} \sum_{i=1}^{3}\left(V_{u_{i} d} V_{d u_{i}}^{\dagger}\right)\right)\right. \\
& \left.+\frac{1}{2 m_{W}^{2} s_{w}^{2}}\left(\frac{1}{2} \sum_{i=1}^{3}\left(V_{u_{i} d} V_{d u_{i}}^{\dagger} m_{u_{i}}^{2}\right)+m_{d}^{2}\left(\frac{1}{4}+I_{3 d}^{2}\right)\right)\right] .
\end{aligned}
$$

